\title{
NIVELES DE GLUCOSA Y TRIGLICÉRIDOS EN PLASMA SEMINAL Y MOTILIDAD ESPERMÁTICA EN COBAYOS ALIMENTADAS CON 10\% MÁS EN ENERGÍA DIGESTIBLE
}

\section{LEVELS OF GLUCOSE AND TRIGLYCERIDES IN SEMINAL PLASMA AND SPERMATIC MOTILITY IN GUINEA PIG FED WITH 10\% MORE IN DIGESTIBLE ENERGY}

\section{Rodríguez G, J. ${ }^{1 *}$, Barrios-Arpi, M. ${ }^{2}$, Huanca L, W. ${ }^{3}$, Rodríguez G, A. ${ }^{4}$, Revuelta, L ${ }^{5}$. \\ ${ }^{1}$ Estación Experimental-IVITA, Mantaro, ${ }^{2}$ Laboratorio de Patología Clínica y Biología Molecular, ${ }^{3}$ Laboratorio de Reproducción Animal- Facultad de Medicina Veterinaria- Universidad Nacional Mayor de San Marcos. ${ }^{4}$ SENASA, Región Junín-Perú \\ ${ }^{5}$ Departamento de Fisiología Animal, Universidad Complutense de Madrid-España *Correspondencia al autor: jrodriguezg3@unmsm.edu.pe}

\section{RESUMEN}

El objetivo del presente estudio fue determinar los niveles de los componentes energéticos (glucosa y triglicéridos) en el plasma seminal y la motilidad espermática de 10 cobayos, los cuales fueron alimentados por un período de 2 meses con una dieta $10 \%$ mayor en energía digestible (ED). El nivel de glucosa encontrado en plasma seminal fue de $11.59 \pm 0.5 \mathrm{mg} / \mathrm{dL}$ y el valor de triglicéridos fue de $55.95 \pm 3.2 \mathrm{mg} / \mathrm{dL}$, mientras que la motilidad promedio fue de 95\%. Concluimos que en cobayos los niveles de glucosa y triglicéridos se incrementan al aumentar el nivel de ED en dieta sin producir alteraciones en la motilidad espermática.

Palabras clave: cobayos, plasma seminal, glucosa, triglicéridos, motilidad espermática. 


\begin{abstract}
The objective the study was to determine the levels of glucose and triglycerides in seminal plasma of 10 guinea pigs, which were fed for a period of 2 months with a diet containing $10 \%$ more ED. The level of glucose found in seminal plasma was $11.59 \pm 0.5 \mathrm{mg} / \mathrm{dL}$ and triglyceride value was $55.95 \pm 3.2 \mathrm{mg} / \mathrm{dL}$, while the motility was $97 \%$ on average. We conclude that in guinea pigs the levels both glucose and triglycerides were increased by major level of ED in feed, but the spermatic motility was not.
\end{abstract}

Key words: guinea pig, seminal plasma, glucose, triglycerides, spermatic motility.

\title{
INTRODUCCIÓN
}

El plasma seminal, el cual es una mezcla compleja secretada de los testículos, epidídimo y glándulas accesorias, puede afectar la morfología espermática, motilidad, reacción acrosomal y la fertilidad (Mann y Lutwak-Mann, 1981); además de su importancia fisiológica, la composición molecular es compleja y la diversidad de proteínas o polipéptidos esta pobremente entendida. Se sugiere que la composición proteica del plasma seminal es diferente entre especies y que algunas proteínas plasmáticas están asociadas con la fertilidad en varias especies. Asimismo, se sabe que la calidad del semen, se mide a través de la motilidad, vialidad, test hipoosmolar, concentración y morfología espermática, éstas variables determinan de forma clásica dicha calidad (Hafez y Hafez, 2000). Otra forma de incrementar las variables que permitan reconocer una buena calidad de semen sería por lo métodos bioquímicos, como por ejemplo niveles de fructosa, glucosa, lípidos, iones y otros componentes presentes en el plasma seminal.

Los componentes químicos presentes en el plasma seminal tienen diferentes funciones, entre las que se encuentran la de proveer energía a los espermatozoides por medio de la fructuosa, glucosa y lípidos (Soengas et al., 1993, Lahnsteiner et al., 1993). Así también, el plasma seminal contiene sustancias que le dan soporte a las células espermáticas, tales como sodio y potasio que estabilizan el balance osmótico, elementos traza como componentes de importantes enzimas (Zamiri y Khodaei, 2005), así como el calcio, este último, necesaria para la estimulación de la esteroidogénesis en células de leydig de los testículos (Henricks, 1991) 
La composición de azúcares del plasma seminal se ha relacionado con la fertilidad, debido a su importancia, principalmente para la producción de energía para los espermatozoides (Willians y Ford, 2001). La fructosa, que es el azúcar principal del plasma seminal, se origina a partir de las vesículas seminales y es esencial para el metabolismo y motilidad espermática (Lewis-Jones et al., 1996). Otros componentes bioquímicos, como el colesterol, tienen como función la orientación reguladora y el flujo de la membrana lipídica (Cross, 1998). Aparte de la interacción con polisacáridos, fosfolípidos, lipoproteínas, iones bivalentes, las proteínas del plasma seminal reconocen receptores del plasmalema del esperma y la zona pelúcida (Mogielnicka-Brzozowska y Kordan, 2011).

Las mitocondrias del espermatozoide pueden metabolizar los monosacáridos completamente a $\mathrm{CO}_{2}$ y agua por la combinación de glucólisis, fructólisis y por la actividad del ciclo de Krebs. Las mitocondrias espermática son las únicas que contienen lactato deshidrogenasa, lo que la hace capaz de utilizar el lactato, producto del metabolismo, en la obtención de más energía (Mansour et al., 2002).

Con relación al papel que cumplen las enzimas, la lactato deshidrogenasa (LDH), la aspartato amino transferasa (AST) y la fosfatasa alcalina (ALP), son esenciales para los procesos metabólicos que proveen energía para la sobrevivencia, motilidad y fertilidad de los espermatozoides (Turner y McDonnell, 2003).

El presente estudio tuvo por objetivo incrementar los niveles energéticos en plasma seminal sin que esto afecte la motilidad espermática, tras la suplementación de una dieta alta en energía (10\% más ED) y así tener la posibilidad de extrapolar los resultados obtenidos para el caso de los humanos dándole la importancia al cobayo como modelo experimental.

\section{MATERIAL Y MÉTODO}

Se utilizaron cobayos de 2 meses de edad. 4 cobayos control con pienso estándar comercial, y 10 cobayos tratadas con un pienso $10 \%$ mayor en ED. Fueron criados en batería por un periodo de 2 meses en las instalaciones del Laboratorio de Farmacología y Fisiología de la Facultad de Medicina Veterinaria, Universidad Nacional Mayor de San Marcos, Lima. Se les suministró durante este periodo pienso contenía $3 \mathrm{Mcal} / \mathrm{kg}$ al control y $3,3 \mathrm{Mcal} / \mathrm{Kg}$ a los tratados, $13 \%$ proteínas, $2 \%$ grasas, $16 \%$ fibra, $12 \%$ humedad, $9 \%$ cenizas, $0.5 \%$ fósforo y $0.5 \%$ de calcio y agua ad-libitum, las condiciones de temperatura se mantuvieron en un rango de 22- 
$24^{\circ} \mathrm{C}$. Al término de la crianza fueron sacrificados por sobredosis de pentobarbital sódico, luego se procedió a realizar una incisión en la región abdominal y pélvica donde se expusieron las gónadas y glándulas accesorias. Del epidídimo se extrajo muestras de espermatozoides, y a continuación se evaluó la motilidad (\%); mientras que el plasma seminal se obtuvo directamente de la vesícula seminal, el que fue centrifugado a 3500 r.p.m por 40 minutos, del sobrenadante se determinó los niveles de glucosa y triglicéridos, para lo cual se tomó $10 \mathrm{uL}$ y se mezcló por 5' con $1 \mathrm{~mL}$ de reactivo de trabajo del kit de glicemia enzimática AA4 (Wiener Lab., Argentina) a $37^{\circ} \mathrm{C}$ y con $10 \mathrm{uL}$ del mismo sobrenadante se mezcló con $1 \mathrm{~mL}$ de kit para triglicéridos (FAR Diagnostics, Italia) y se incubó a $37^{\circ} \mathrm{C}$, después se realizó la lectura en el Analizador Bioquímico Sinnowa Medical Science BS3000M (Nanjing, China). Los valores obtenidos fueron expresados en $\mathrm{mg} / \mathrm{dL}$ y expresados en promedio \pm desviación estándar. Para determinar las diferencias estadísticas significativas se usó T-Student con un nivel de confianza del 95\%.

\section{RESULTADOS Y DISCUSIÓN}

En el presente estudio, fueron investigados algunos de los componentes el plasma seminal y su correlación con la motilidad espermática en el cobayo como modelo experimental.

Tabla 1. Niveles de glucosa y triglicéridos en plasma seminal de cobayos con $10 \%$ mayor ED en pienso (promedio \pm desviación estándar).

Control

Parámetro bioquímico
Tratado

\begin{tabular}{lllll}
\hline Glucosa (mg/dL) & 10.02 & 0.9 & $11.59^{*}$ & 0.5 \\
Triglicéridos (mg/dL) & 48.33 & 2.4 & $55.95^{*}$ & 3.2 \\
\hline
\end{tabular}

*Diferencia estadística significativa ( $p<0.05)$. DS: desviación estandar

Como podemos ver en la Tabla 1, en el presente estudio hemos podido encontrar niveles de glucosa de $11.59 \mathrm{mg} / \mathrm{dL}$ y de triglicéridos de $55.95 \mathrm{mg} / \mathrm{dL}$ en animales tratados, valores mayores al respectivo control. Asimismo, la motilidad mostrada por los espermatozoides en 
animales tratados supera al control pero no es significativo. La concentración de diversos componentes bioquímicos en el plasma seminal difiere entre especies. En otros estudios, donde además de evaluar las características espermáticas evaluaron la bioquímica del plasma seminal humano encontrando niveles de glucosa de 39,50 mg/dL (Igbinaduwa e Igbinaduwa, 2007) y $47.17 \mathrm{mg} / \mathrm{dL}$ (Truta et al., 2010) y una motilidad de hasta el 100\% (Igbinaduwa e Igbinaduwa, 2007) en individuos normales; mientras que en conejos los niveles de lípidos totales (entre los que se encuentra los triglicéridos) fue de $130 \mathrm{mg} / \mathrm{dL}$ y la motilidad espermática tuvo un valor de 74.2\% (Attia et al., 2010), en camellos, la glucosa de plasma seminal fue de $35.8 \mathrm{mg} / \mathrm{dL}$, triglicéridos de $101.6 \mathrm{mg} / \mathrm{dL}$ y la motilidad de 62.4\% (Mosaferi et al., 2005); en búfalos, los niveles de colesterol se encontraban en el rango de 41 a $48 \mathrm{mg} / \mathrm{dl}$ y colesterol total de 52 a 55 $\mathrm{mg} / \mathrm{dl}$ (Shukla et al., 2009); mientras que en peces como la carpa plateada los niveles de glucosa son bastante bajos y van desde 1.2-1.8 mg/dL, los triglicéridos van de 5-12 mg/dL y la motilidad es de 60-75\% (Faramarzi, 2012); en carpa escamosa la glucosa fue de $1.43 \mathrm{mg} / \mathrm{dL}$, los triglicéridos $7.82 \mathrm{mg} / \mathrm{dL}$ y la motilidad de $63.18 \%$ (Bozkurt et al., 2009). Un estudio reportado por Cevik et al. (2007) demostró que los niveles de calcio y fósforo en toros oligoastenozoospérmicos eran menores a los encontrados en animales normozoospérmicos; y asimismo determinó que los niveles de glucosa en plasma seminal eran significativamente más elevados en toros normozoospérmicos que en oligoastenozoospérmicos. Asimismo, se han reportado los niveles de calcio, GPT y GOT en búfalos, hallándose los siguientes rangos 6-16 mg/dl, 2-21 UI/L y 85-561 UI/L, respectivamente (Barnabe et al., 1993).

Los carbohidratos y los lípidos son las principales fuente de energía utilizadas para el trabajo celular, entre éstos, encontramos a los monosacáridos como la glucosa y lípidos como los triglicéridos que se encuentran en plasma y de allí pueden pasar al extracelular de diferentes células para que estas las puedan usar como "combustible" (Berg et al., 2007). La presencia de este azúcar en el plasma seminal se debe a la alta demanda de energía de los testículos durante la espermatogénesis o para la síntesis de lípidos de los espermatozoides (Soengas et al., 1993). Por su parte, los triglicéridos sirven como recursos de energía para los espermatozoides inmóviles durante el almacenamiento y durante la fase de regeneración después de la motilidad (Lahnsteiner et al., 1993). Mientras que los bajos niveles de triglicéridos, podría ser indicativo de la insuficiencia de los recursos energéticos, la tasa de reducción de la motilidad y la capacidad de fertilización (Lahnsteiner et al. 1994).

La motilidad espermática no mostró diferencias significativas en los animales que fueron tratados con aumento del $10 \%$ de energía digestible (Tabla 2), por ello no se 
incrementaría la motilidad espermática por un leve aumento de la energía digestible en la dieta; sin embargo es probable que por un aumento gradual se puedan observar cambios significativos en la motilidad espermática y así poder extrapolar estos resultados en humanos utilizando al cuy como modelo experimental.

Tabla 2. Motilidad espermática (\%).

\begin{tabular}{cccc}
\hline & \multicolumn{3}{c}{ Motilidad (\%) } \\
\cline { 2 - 4 } & Mínima & Máxima & Promedio \\
\hline Control & 92 & 98 & 95 \\
\hline Tratado & 94 & 99 & 97 \\
\hline
\end{tabular}

La correlación entre proteínas del plasma seminal y la fertilidad del macho ha sido reportado en algunas especies de animales domésticos tales como toros (Killian et al., 1993), carneros (Jobim et al., 2005), caprinos (Villemure et al., 2003), búfalos (Asadpour et al., 2007), sementales y jabalíes (Calvete et al., 1999). Sin embargo, no se ha podido demostrar en estudios previos si existiría una correlación entre los niveles de glucosa, fructosa, triglicéridos y colesterol en especies como los cobayos.

\section{CONCLUSIONES}

En cobayos, los niveles de glucosa y triglicéridos se elevaron significativamente por efecto de un pienso con $10 \%$ más de energía digestible y aunque la motilidad fue mayor, no resultó estadísticamente significativa.

\section{BIBLIOGRAFIA}

Asadpour R, Alavi-Shoushtari SM, Asri Rezaii S, Ansari MHKH. 2007. SDS-polyacrylamide gel electrophoresis of buffalo bulls seminal plasma proteins and their relation with semen freezability. Animal Reproduction Science 102(3-4): 308-313.

Berg J, Tymoczko J, Stryer L. 2007. Bioquímica. 6ª ed. Barcelona: Reverté. 1026p. 
Barnabe RC, Barnabe VH, De Oliveira CA, De Arruda RP, Valentim R, Zogno MA. 1993. Biochemical study of semen of buffaloes. I. determinations of frutose, calcium, GOT and GPT. Braz J Vet Res Anim Sci 30(1): 43-45.

Bozkurt Y, Ogretmen F, Secer FS, Ercin U. 2009. Relationship Between Seminal Plasma Composition and Spermatological Parameters in Scaly Carp (Cyprinus carpio). Journal of Animal and Veterinary Advances.8 (12): 2745-2749.

Calvete JJ, Campanero-Rhodes MA, Raida M, Sanz L. 1999. Characterization of the conformational and quaternary structure-dependent heparin-binding region of bovine seminal plasma protein PDC-109. FEBS Lett 444(2): 260-264.

Cevik M, Tuncer PB, Tasdemir U, Ozgurtas T, 2007. Comparison of Spermatological Characteristics and Biochemical Seminal Plasma Parameters of Normozoospermic and Oligoasthenozoospermic Bulls of Two Breeds.Turk. J Vet Anim Sci 31: 381-387.

Cross NL. 1998. Role of cholesterol in sperm capacitation. Biol Reprod 59: 7-11.

Faramarzi M. 2012. Assessment of Reproductive Parameters in Silver Carp (Hypophthalmichthys molitrix). World Journal of Fish and Marine Sciences.4 (3): 244-248.

Hafez ESE, Hafez B. 2000. Reproducción e inseminación artificial en animals. $7^{\mathrm{a}}$ ed. USA: McGraw Hill. 519p.

Henricks DM. 1991. Biochemistry and Physiology of the Gonadal Hormones. In: Cupps P.T. (Ed). Reproduction in Domestic animals. 4th edn. San Diego: Academic Press. 670p.

Igbinaduwa P, Igbinaduwa B. 2007. The Predictive Value of Glucose-fructose Ratio in Seminal Plasma. World Applied Sciences Journal 2 (6): 602-605.

Jobim MI, Oberst ER, Salbego CG, Wald VB, Horn AP, Mattos RC. 2005. BSP A1/A2-like proteins in ram seminal plasma. Theriogenology 63(7): 2053-2062.

Killian GJ, Chapman DA, Rogowski LA. 1993. Fertility-associated proteins in Holstein bull seminal plasma. Biol. Reprod 49: 1202-1207.

Lahnsteiner F, Patzner R, Weismann T. 1994. The testicular main duct and the spermatic duct in some cyprinid fishes II. Composition of the seminal fluid. J. Fish Biol. 44:459-467.

Lahnsteiner F, Patzner RA, Weismann T. 1993. Energy resources of spermatozoa of the rainbow trout, Oncorhynchus mykiss. (Pisces, Teleostei). Reprod. Nutr. Dev. 33:349-360.

Lewis-Jones DI, Aird IA, Biljan MM. 1996. Effects of sperm activty on zinc and fructose concentrations in seminal plasma. Human Reprod. 11: 2465-2467.

Mann T, Lutwak-Mann C. 1981. Male Reproductive Function and Semen. Berlin: SpringerVerlag. 495p. 
Mansour N, Lahnsteiner F, Patzner RA. 2002. The spermatozoon of the African catfish: Fine structure, motility, viability and its behavior in seminal vesicle secretion. J.Fish Biol. 60: $545-560$.

Mogielnicka-Brzozowska M, Kordan W. 2011. Characteristics of selected seminal plasma proteins and their application in the improvement of the reproductive processes in mammals. Pol J Vet Sci 14:489-499.

Mosaferi S, Niasari-Naslaji A, Abarghani A, Gharahdaghi AA, Gerami A. 2005. Biophysical and biochemical characteristics of bactrian camel semen collected by artificial vagina. Theriogenology. 63(1): 92-101.

Shukla MK, Misra AK, Gupta HP. 2009. Studies on some biochemical constituents of Murrah buffalo (Bubalus bubalis) seminal plasma. Buff Bull 28: 123-126.

Soengas JL, Sanmartin B, Barciella P, Aldegunde M, Rozas G. 1993. Changes in carbohydrate metabolism in domesticated rainbow trout Oncorhynchus mykiss related to spermatogenesis. Comp. Biochem. Physiol. 105: 665-671.

Truta Z, Garlovanu M, Lerintiu S, Micu R. 2010. A new method for human semen glucose concentration evaluation. Romanian Biotechnological Letters. 15 (6): 5764-5772.

Turner RM, McDonnell SM. 2003. Alkaline phosphatase in stallion semen: haracterization and clinical applications. Theriogenology 60(1): 1-10.

Villemure M, Lazure C, Manjunath P. 2003. Isolation and characterization of gelatin-binding proteins from goat seminal plasma. Reproductive Biology and Endocrinology 39 (1): 39-50.

Williams AC, Ford WC. 2001. The role of glucose in supporting motility and capacitation in human. J. Androl.22: 680-695.

Youssef A Attia, A E Abd El Hamid, Fulvia Bovera, Mohamed El-Sayed. 2010. Oral glucose supplementation improved semen quality and constituents of seminal and blood plasma of NZW buck rabbits in the subtropics. Open Access Animal Physiology. 2: 81-85.

Zamiri MJ, Khodaei HR. 2005. Seasonal thyroidal activity and reproductive characteristics of Iranian fat-tailed rams. Animal Reproduction Science. 88(3-4): 245-255. 